\title{
Superoutbursts, superhumps and the tidal-thermal instability model
}

\author{
V. Buat-Ménard and J.-M. Hameury \\ UMR 7550 du CNRS, Observatoire de Strasbourg, 11 rue de l'Université, 67000 Strasbourg, France \\ Received 6 November 2001 / Accepted 19 February 2002

\begin{abstract}
We include the tidal instability due to the 3:1 resonance in the disc instability model developed by Hameury et al. (1998) and modified by Buat-Ménard et al. (2001). We confirm earlier results by Osaki (1989) that the tidal instability can account for the SU UMa light curves. We show that in ultra-low mass ratio systems such as ER UMa stars and WZ Sge stars, the superoutburst ends while the disc is still eccentric, as proposed by Hellier (2001). However, since the disc shrinks rapidly once a cooling wave has started, the eccentricity should stop shortly after the end of a superoutburst. This result disagrees with the suggestion by Hellier that decoupling the thermal and tidal instability in the TTI model can account for late superhumps and echo outbursts in ultra-low mass ratio systems. We propose instead that ER UMa short supercycles can be explained either by the alternation of narrow and wide outbursts similar to those occurring in SS Cyg, or by the effects of irradiation (Hameury et al. 2000). In both cases, we predict that superhumps should be permanent, which is suggested by observations (Gao et al. 1999). We can also reproduce light curves similar to those of EG Cnc, varying the mass transfer rate in a TTI model including both irradiation and the presence of an inner hole in the disc.
\end{abstract}

Key words. accretion, accretion discs - instabilities - stars: novae, cataclysmic variables - stars: binaries: close

\section{Introduction}

Dwarf novae are cataclysmic variables $(\mathrm{CVs})$ which exhibit 2-6 mag recurrent outbursts (see Warner 1995 for an encyclopaedic review). It is generally accepted that these outbursts are due to a thermal-viscous instability that occurs in an accretion disc in which the viscosity is given by the $\alpha$-prescription (Shakura \& Sunyaev 1973). The instability arises when hydrogen becomes partially ionized and the opacities vary steeply with temperature; this occurs when the disc material reaches temperatures of the order of $8000 \mathrm{~K}$ (see Lasota 2001 for a review of the model). A number of characteristics of many light curves (characteristic time scales, amplitudes, ...) are reproduced by the disc instability model (DIM) provided the viscosity parameter $\alpha$ is different in quiescence and in outburst. However several subclasses of dwarf novae show specific features that are not easy to explain with the standard version of the DIM.

SU UMa stars are defined as dwarf novae in which normal outbursts cycles are regularly interrupted by anomalously bright and long outbursts called superoutbursts. These outbursts are 0.7 mag brighter and last 5 to 10 times

Send offprint requests to: V. Buat-Ménard, e-mail: buat@astro.u-strasbg.fr longer than the normal ones. The mean time $T_{\mathrm{s}}$ between two consecutive superoutbursts defines the period of a supercycle $T_{\mathrm{s}}$; in most SU UMa stars, $130<T_{\mathrm{s}}<350$ days. There are however two extreme exceptions: ER UMa stars have anomalously short supercycles (between 19 and 45 days), while WZ Sge stars show only superoutbursts separated by very long quiescence periods that can reach 30 years. In addition, the light curve is modulated during superoutbursts at a period slightly longer than the orbital period. These so-called superhumps usually disappear shortly after the decline of the superoutburst. Whitehurst (1988) explained these superhumps by the precession of a distorted disc. The name "superoutburst" is reserved for dwarf nova eruptions in which a superhump is present. This property differentiates superoutbursts from wide outbursts observed in many dwarf novae; van Paradijs (1983) suggested that superoutbursts are just wider outbursts in the bimodal outburst-length distribution. In addition, a 1985 outburst of U Gem had all the properties of a superoutburst (of WZ Sge stars) except for the superhump (see Kuulkers et al. 1999 and references therein). This could suggest that the superoutburst phenomenon is independent of the disc distortion. We discuss this possibility in Sect. 4 . 
As almost all SU UMa stars have periods below the period gap of CVs, they all have low secondary to primary mass ratio $q$, and the primary Roche lobe is large. The disc can therefore extend to relatively large radii, and this led Osaki (1989) to propose the thermal-tidal instability (TTI), in which superoutbursts are caused by the disc reaching the 3:1 resonance radius, as a model for SU UMa stars. SPH simulations by Whitehurst (1988) showed that the disc becomes eccentric and precesses when its radius reaches the 3:1 resonance radius, but it must be kept in mind that the hydrodynamical simulations of Stehle (1999) and Kornet \& Różyczka (2000) did not show such an effect.

Detailed observations of SU UMa stars point out several limitations of the TTI model. The mass transfer rate from the secondary is observed to be enhanced during outburst; this increase, possibly due to irradiation, is not taken into account, although it can drastically alter the light curves (Hameury et al. 2000).

The TTI model cannot easily reproduce the properties of ER UMa and WZ Sge subclasses. In order to obtain the very short ER UMa supercycles (19 to 45 days), one must assume that the tidal instability shuts off prematurely (Osaki 1995a) for very small mass ratios. The very long quiescence time of WZ Sge systems would on the other hand require a very small viscosity in quiescence (Smak 1993).

Occasionally, some systems show echo outbursts at the end of a superoutburst: several consecutive small outbursts with very short recurrence time are triggered before the disc returns to quiescence. During these echooutbursts, superhumps are still present. These so-called late superhumps (LS) have also been observed in ER UMa stars. However, the TTI model simulations predict that the end of a superoutburst should coincide with the cessation of the tidal instability.

These different problems have lead several authors to investigate alternative possibilities. Hameury et al. (2000) have shown that if one takes into account the disc and secondary illumination in the standard DIM, one is able to reproduce light curves reminiscent of those of systems such as RZ LMi or EG Cnc. Recently Hellier (2001) suggested that some difficulties could be solved in the framework of a slightly modified TTI model; he proposed that the superoutburst must end before the end of eccentricity in low mass ratio systems, thus giving an explanation for LS.

We have included in our DIM model (Hameury et al. 1998; Buat-Ménard et al. 2001, hereafter Paper I) an enhanced tidal torque when the disc radius exceeds the 3:1 resonance radius. We do reproduce the earlier results of Osaki (1989), obtained using a simplified model. We show that in a number of cases, a cooling wave can end a superoutburst while the disc is still eccentric, thereby confirming the proposal of Hellier (2001). However, this by itself is not sufficient to reproduce the properties of ER UMa or WZ Sge systems, and additional effects such as illumination and mass transfer variations must be included.

\section{The TTI model}

The equation of angular momentum conservation in a disc is (Hameury et al. 1998):

$$
\begin{aligned}
j \frac{\partial \Sigma}{\partial t}=-\frac{1}{r} \frac{\partial}{\partial r}\left(r \Sigma j v_{\mathrm{r}}\right) & +\frac{1}{r} \frac{\partial}{\partial r}\left(-\frac{3}{2} r^{2} \Sigma \nu \Omega_{\mathrm{K}}\right) \\
& +\frac{j_{2}}{2 \pi r} \frac{\partial \dot{M}_{\mathrm{tr}}}{\partial r}-\frac{1}{2 \pi r} T_{\mathrm{tid}}(r)
\end{aligned}
$$

where $\Sigma$ is the surface column density, $\dot{M}_{\text {tr }}$ is the rate at which mass is incorporated into the disc at radius $r$, $v_{\mathrm{r}}$ is the radial velocity in the disc, $j=\left(G M_{1} r\right)^{1 / 2}$ is the specific angular momentum of material at radius $r$ in the disc, $\Omega_{\mathrm{K}}=\left(G M_{1} / r^{3}\right)^{1 / 2}$ is the Keplerian angular velocity (where $M_{1}$ is the primary mass), $\nu$ is the kinematic viscosity coefficient, and $j_{2}$ the specific angular momentum of the material transferred from the secondary. $T_{\text {tid }}$ is the torque due to the tidal forces, for which we use the formula of Smak (1984), derived from the determination of tidal torques by Papaloizou \& Pringle (1977):

$T_{\mathrm{tid}}=c \omega r \nu \Sigma\left(\frac{r}{a}\right)^{5}$

where $\omega$ is the angular velocity of the binary orbital motion, $a$ the binary orbital separation and $c$ a numerical constant taken so as to give an average disc radius equal to some chosen value.

In the TTI model, $c$ is no longer a constant, but depends on whether the disc is eccentric or not. Ichikawa et al. (1993) reproduced supercycles assuming that the disc becomes eccentric when its radius reaches $r_{3: 1} \sim$ $0.46 a ; c$ is then increased by a large factor. Afterward the disc starts shrinking and can no longer maintain its eccentricity when its radius becomes smaller than $r_{\text {crito }} \sim 0.35 a$, at which point $c$ returns to its normal value. We choose to take a value $c_{0}$ when the disc is axisymmetric and a larger value $c_{1}$ when the disc is eccentric. This is our Prescription $I$ for $c$, quite similar to that of Ichikawa et al. (1993) who extended the work of Osaki (1989) by taking into account a non zero tidal torque during quiescence.

We have included this variable tidal torque in the disc instability model used in Paper I, which is a modified version of the DIM described in Hameury et al. (1998) that includes heating by the stream impact and the tidal torque (we have corrected Eq. (1) of Paper I, in which the heating rate was overestimated by a factor $\sim 2$ ). In the following, we also assume that $\alpha=\alpha_{\text {cold }}=0.04$ in quiescence and $\alpha=\alpha_{\text {hot }}=0.2$ in outburst. The inner radius is fixed at $r_{\text {in }}=10^{9} \mathrm{~cm}$.

We obtain the cycle shown in Fig. 1. After a superoutburst, the disc mass and radius are small. The disc mass slowly increases, and several normal outbursts are triggered due to the thermal instability. During each outburst, only a small amount of mass is accreted by the white dwarf, so that the average disc mass increases; a significant disc expansion occurs during the rise to maximum, when the heat front reaches the outer edge of the disc. The disk then shrinks when a cooling front brings the 


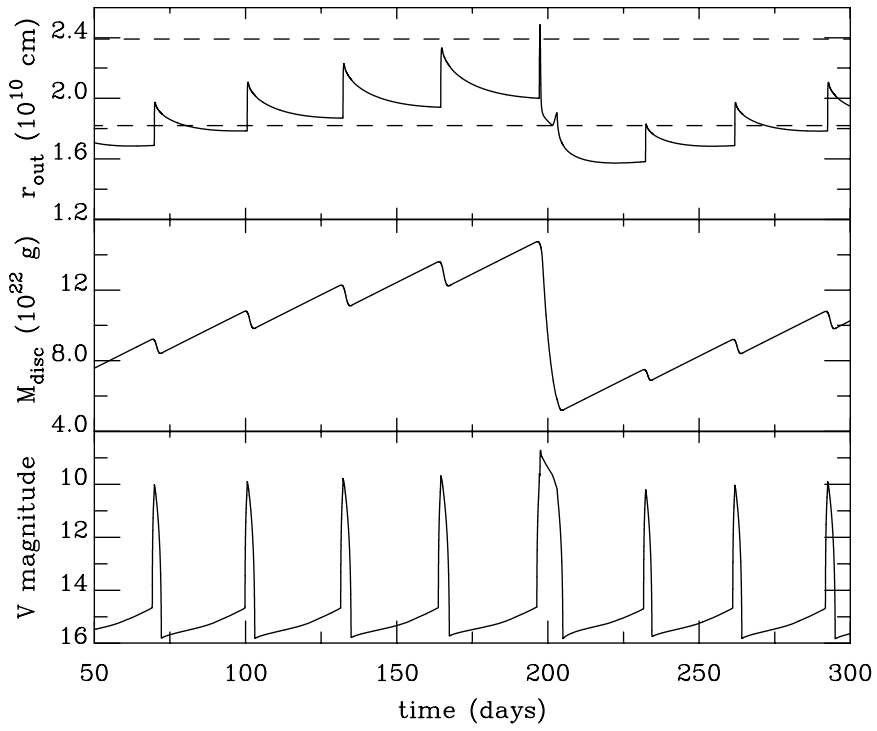

Fig. 1. Superoutburst cycle in the TTI model for SU UMa with $\dot{M}_{\mathrm{tr}}=10^{16} \mathrm{~g} \mathrm{~s}^{-1}$. Top panel: disc radius; intermediate panel: disc mass; bottom panel: disc visual magnitude.

disc back to a cool state, causing a significant reduction of the outward angular momentum flow. After the end of an outburst, the disc radius very slowly decreases as a result of mass addition and viscous diffusion, but the net balance is an increase of the radius from one outburst to the next one. Eventually, $r_{\text {out }}$ becomes larger than $r_{3: 1}$ during an outburst and the tidal torque is increased, as well as the associated energy dissipation. The disc is then maintained in the hot state until most of the mass has been accreted. During this phase the radius decreases until $r<r_{\text {crit0 }}$. The disc returns to a circular shape, the anomalous tidal dissipation stops, and a cooling wave starts from the disc outer edge, bringing the whole disc in a cool state.

\subsection{Radial dependence of $c$}

There is, however, no compelling reason to assume that the whole disc is affected by the development of the tidal instability. We therefore use another prescription for $c$ (hereafter Prescription II). We assume that when the disc is circular, $c=c_{0}$; when eccentric, $c(r)=c_{1}$ for $r>0.9 r_{\text {crit0 }}$ and $c=c_{0}$ for $r<0.8 r_{\text {crit0 }}$. A linear interpolation is assumed between $0.8 r_{\text {crit0 }}$ and $0.9 r_{\text {crit0 }}$. We compare and discuss the results obtained using both prescriptions in 3.1 .

\subsection{Time delays}

Temporal variations of $c(r)$ are not instantaneous. Observations show that normal superhumps appear within a few of days after the onset of a superoutburst, and that they develop within a day, so that it does not take long for the disc to become eccentric (Semeniuk 1980). However, it takes the disc at least a dynamical time to change its geometrical shape; we assumed that, when the tidal
Table 1. Parameters used for SU UMa subclasses simulations. $M_{2}$ is the secondary mass and $r_{\text {circ }}$ the circulation radius.

\begin{tabular}{lccc}
\hline \hline & SU UMa & \multicolumn{2}{c}{ ULMR Stars } \\
& & ER UMa type & WZ Sge type \\
\hline$M_{1} / M_{\odot}$ & 0.8 & 1.0 & 0.6 \\
$M_{2} / M_{\odot}$ & 0.15 & 0.1 & 0.1 \\
$P_{\text {orb }}(\mathrm{hr})$ & 1.83 & 1.522 & 1.3752 \\
$a / 10^{10} \mathrm{~cm}$ & 5.2 & 4.82 & 3.876 \\
$r_{\text {circ }} / 10^{10} \mathrm{~cm}$ & 0.91 & 1.1 & 0.74 \\
\hline
\end{tabular}

instability sets in, $c$ increases linearly on a timescale $t_{\mathrm{r}}=2 \times 10^{4} \mathrm{~s}$. Note that in WZ Sge systems, the early superhumps that first appear seem to be of a different nature than the normal superhumps; Osaki \& Meyer (2002) suggested that they could be related to the $2: 1$ resonance.

Similarly, the disc must dissipate its excess energy in order to return to a circular shape. The relative energy difference between a circular and an elliptic orbit with eccentricity $e$ with the same angular momentum is $\Delta E / E_{\text {circ }}=$ $e^{2}$. As the dissipation at radius $r$ is $D \sim G M \Sigma \nu / r^{3}$, the time needed for the disc to return to a normal state is:

$t_{\mathrm{e}-\mathrm{c}}=\frac{\Delta E}{D}=e^{2} \frac{r^{2}}{2 \nu}=e^{2} t_{\nu}$

where $t_{\nu}$ is the viscous time in the outer part of the disc. When the disc reverts to its normal state, the cooling wave has already started or will start with the end of tidal instability (see Sect. 3). $t_{\nu}$ therefore refers to the cool state, and is of the order of the recurrence time, since the outbursts obtained here are of the inside-out type. Typical values are tens of days. Numerical simulations of the development of the tidal instability show that $e$ is of order of 0.1-0.3 (Murray 1996), which is confirmed by observations (Patterson et al. 2000), and $t_{\mathrm{e}-\mathrm{c}}$ should be a few percent of $t_{\nu}$. We then assume that $c$ linearly decreases when the disc returns to a circular shape on a time $t_{\mathrm{f}}=2 \times 10^{5} \mathrm{~s}$.

We use three different sets of parameters that are representative for SU UMa, a typical ER UMa star and a typical WZ Sge star respectively, shown in Table 1. $r_{\text {circ }}$ is the circulation radius at which a particle leaving the Lagrangian point would stay in circular orbit if there were no accretion disc (see Paper I for details). One should note that $r_{\text {circ }}$ is calculated taking into account the gravitational potential of the secondary, and therefore differs slightly from the Keplerian value. In the following section, we will use the term ultra-low mass-ratio (ULMR) stars when referring to simulations with ER UMa parameters.

\section{Decoupling the thermal and tidal instabilities}

\subsection{A property of ultra-low mass ratio systems?}

Hellier (2001) suggested that the ultra-low mass ratio of ER UMa and WZ Sge stars is responsible for their peculiarities. In their case, the Roche lobe extends far beyond 
the $3: 1$ resonance radius, and the disc could in principle be larger than $r_{3: 1}$. Hellier proposed that regions outside the resonance radius suffer a lower tidal dissipation than the portions in the 3:1 resonance zone. Then, during a superoutburst, heating by the tidal torque will not be as efficient as in other SU UMa stars, and a cooling wave could start while the disc is still eccentric, and will remain so several days after the end of the superoutburst.

This would then account for late superhumps. In addition, Hellier suggested that this model could also explain the echo outbursts in WZ Sge stars and the short ER UMa supercycle: at the end of a superoutburst, the disc would be still eccentric, and the tidal dissipation sufficiently large to trigger short outbursts during which the heating wave does not propagate to the outer edge (one should add that such outbursts are not only short but also have amplitudes lower than outbursts in which the heating front reaches the outer disc's edge). ER UMa stars would then be systems always in an eccentric state, while WZ Sge stars would finally return to complete quiescence with circular discs. The difference between the supercycle duration of ER UMa and WZ Sge stars would be due to higher mass-transfer rates in ER UMa's.

Figure 1 shows the simulated light curve for the parameters of SU UMa, assuming a factor 20 between $c_{1}$ and $c_{0}$. The superoutburst ends when the disc radius becomes lower than $r_{\text {crito }}$, i.e. the end of the tidal instability causes the end of the superoutburst. Figure 2 shows the simulated light curve for the parameters of ER UMa, with the same $c_{1} / c_{0}$. The mass transfer rate of $10^{16} \mathrm{~g} \mathrm{~s}^{-1}$ chosen here is close to the critical value above which the system is stable (within a factor 1.3). In this case, as predicted by Hellier, the superoutburst ends because the thermal instability stops, while the tidal instability is still effective. However, contrary to Hellier's hypothesis (2001), this phase is very short because of the rapid shrinking of the disc. Therefore, the duration of the supercycle is much larger than observed (there is practically no quiescence phase in real ER UMa stars): short outbursts are due to the usual thermal-viscous instability. As a consequence, contrary to Hellier's assumption, the heating front in short outbursts always reaches the outer disc's edge. Mass transfer rates closer to the stability limit do not lead to shorter supercycles, but rather to longer superoutbursts; this is actually the reason why it is impossible to obtain very short supercycles in the standard TTI model without changing the tidal instability condition (Osaki 1995a).

We have investigated the influence of the ratio $c_{1} / c_{0}$ and of the Roche size on our results. Figure 3 compares the superoutburst behavior for $c_{1} / c_{0}$ factors of 20,50 and 80 for ULMR stars with prescription I. As can be seen, the width of the superoutburst strongly depends on $c_{1}$. Very small and very large values of $c_{1}$ both give short superoutburst, either because the tidal heating is too small (small $c_{1}$ ), or because the disc contraction under the effect of the tidal torque is too large (large $c_{1}$ ). Typical $c_{1} / c_{0}$ should be in the range $\sim 20-50$ in order to reproduce the observed superoutburst durations.

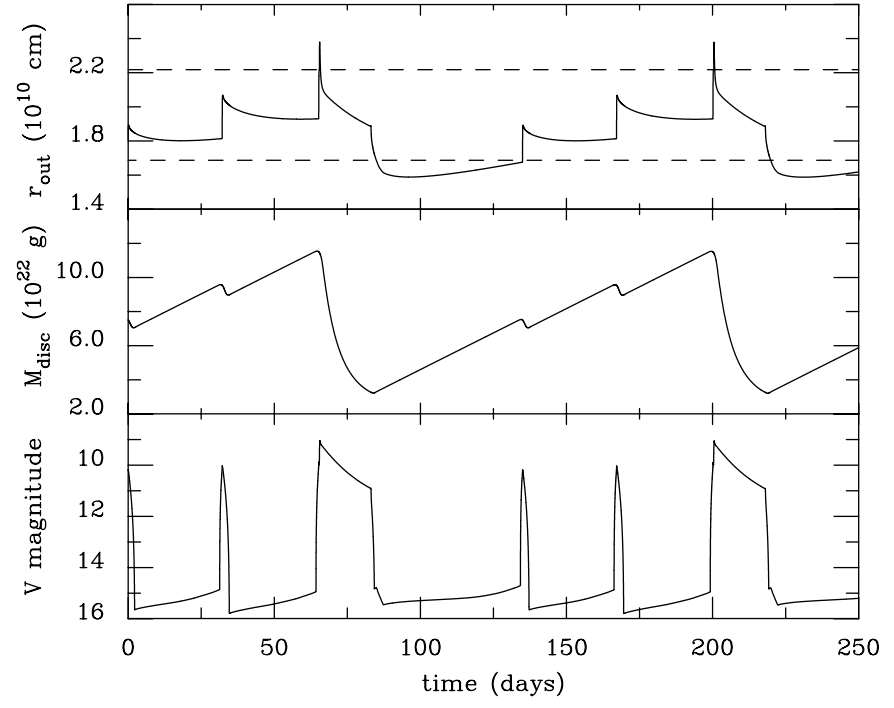

Fig. 2. Same as Fig. 1 for ER UMa parameters.

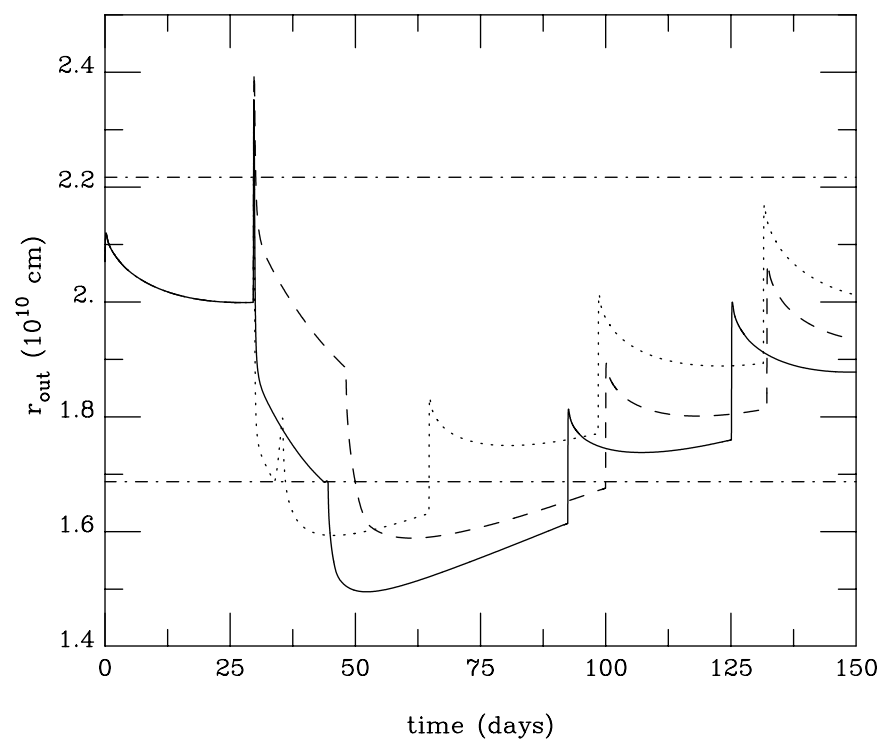

Fig. 3. Radius behavior during superoutburst for ultra-low mass ratio stars with $\dot{M}_{\mathrm{tr}}=10^{16} \mathrm{~g} \mathrm{~s}^{-1}$. The dot-dashed lines represents the $0.46 a$ (upper) and $0.35 a$ (lower) radius. For $c_{1} / c_{0}$ factors of 20 (dashed line) and 50 (solid line), the superoutburst lasts $\sim 21$ days $\left(\sim 17\right.$ days for $\left.c_{1} / c_{0}=50\right)$ and is terminated by a cooling wave. For a factor 80 (solid line) it lasts $\sim 9$ days and ends by the disc becoming circular.

The decoupling of the tidal and thermal instability was found for values of $c_{1} / c_{0}$ lower than 80 , for both prescription I and II for ER UMa parameters, thereby confirming the first part of Hellier (2001) proposal. On the other hand, for the parameters of SU UMa, the instability stopped because the disc shrinks below $0.35 a$, except for $c_{1} / c_{0}<10$; this limit is increased to $\sim 20$ when one uses prescription II.

These results strongly support the idea that the decoupling of thermal and tidal instability is a property of the ultra low mass ratio systems. One should however keep in 
Table 2. Response of the outer disc radius $r_{\mathrm{d}}$ to a decrease of $|\dot{M}|_{\operatorname{tr}}, c$ and $\alpha$ by a factor 2 .

\begin{tabular}{lc}
\hline \hline$x$ & $\mathrm{~d} \log r_{\mathrm{d}} / \mathrm{d} \log x\left(\right.$ at $\left.10^{4} \mathrm{~s}\right)$ \\
\hline$|\dot{M}|_{\mathrm{tr}}$ & $2.5 \times 10^{-2}$ \\
$c$ & $2.67 \times 10^{-3}$ \\
$\alpha$ & $-4.37 \times 10^{-2}$ \\
\hline
\end{tabular}

mind that this conclusion depends somewhat on the assumed radial dependence of $c$, and on the assumed $c_{1} / c_{0}$; extreme values (less than 10 or more than 50) cause too short superoutburst to be triggered but ER UMa stars sometimes have short superoutbursts.

\subsection{The superoutburst cooling wave}

As shown in the previous section, decoupling of tidal and thermal instabilities is expected in ultra low mass ratio SU UMa stars. However, this is not sufficient to explain the late superhumps or echo outbursts observed in ER UMa and WZ Sge systems, as suggested by Hellier (2001). When a cooling front starts to propagate, the radius decreases rapidly (in less than a few days) to its minimum value in the supercycle. If this minimum is less than $r_{\text {crit0 }}$, then the disc will return to a circular shape in a time scale $t_{\mathrm{f}}$, and one does not expect to observe late superhumps. If on the other hand the minimum radius is larger than $r_{\text {crito }}$, the disc always remains eccentric, the superhumps are permanent, but one then loses the very mechanism that was supposed to trigger superoutbursts.

The disc shrinkage at the end of an outburst or a superoutburst is therefore a key question. The radius evolves under the influence of three main effects, as shown in Eq. (1): (i) the addition of mass with low specific angular momentum at the outer edge of the disc and (ii) the tidal torque that both act to contract the disc, and (iii) the viscous outward transport of angular momentum that tends to make the disc larger. These effects are parameterized by $\dot{M}_{\mathrm{tr}}, c$ and $\alpha$ respectively. In order to determine their influence on the disc radius variations, we have changed their values by a factor of 2 (linear variation on a time scale of $10^{4} \mathrm{~s}$ ) for a steady disc in a hot state (Fig. 4). The disc radius in the new steady state is unchanged, except in the case where $c$ is modified; the short term influence of these variations are summarized in Table 2 which shows the value of $\mathrm{d} \log r / \mathrm{d} \log x, 10^{4} \mathrm{~s}$ after the beginning of the variation, $x$ being one of the three parameters. As can be seen, $\mathrm{d} \log r / \mathrm{d} \log c$ is 5 to 10 times smaller than the two other quantities. This explains why $c_{1} / c_{0}$ has to be larger than 10 in order to obtain a superoutburst, and why this ratio needs to be larger than 20 in order for the radius to decrease fast enough during a superoutburst and reach $r_{\text {crit0 }}$ before the cooling wave starts.

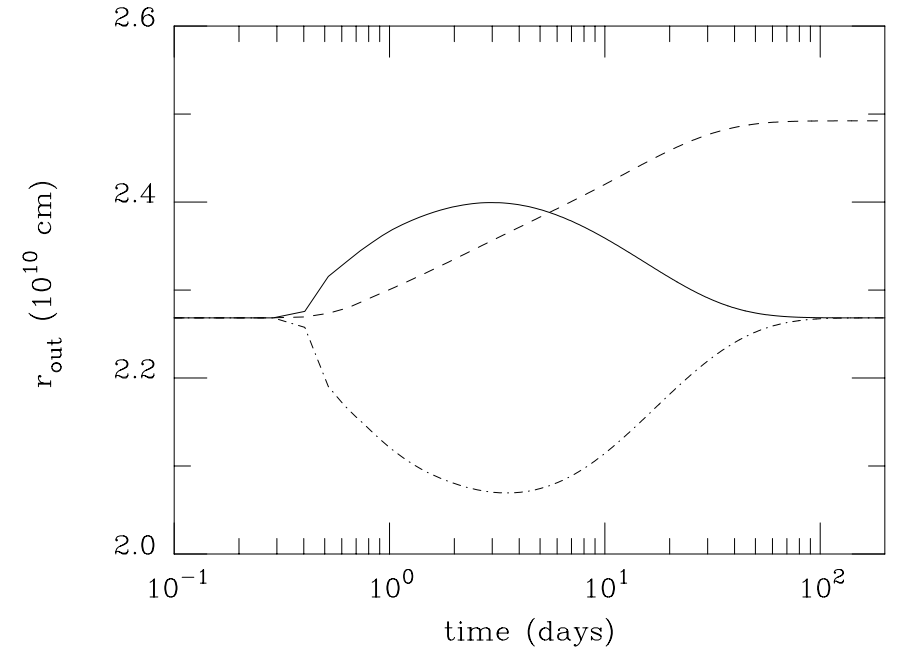

Fig. 4. Responses of the outer radius of a steady accretion disc in the hot state to variation by a factor 2 of $\dot{M}_{\text {tr }}$ (solid line), $c$ (dashed line) and $\alpha$ (dot-dashed line). The parameters vary linearly in $10^{4} \mathrm{~s}$. Note that the perturbation starts at $t \sim 0.3 \mathrm{~d}$.

In principle, the disc shrinkage could be slower, or possibly delayed, if the mass transfer rate from the secondary were reduced, possibly as a result of a decrease in the illumination of the secondary. However, when the cooling wave starts, $\alpha$ decreases from $\alpha_{\text {hot }}$ to $\alpha_{\text {low }}$ in the outer disc regions, i.e. by a factor 5 . $c$ has to be kept fixed, since one requires the disc to remain eccentric. Because of the large change in viscosity when a cooling front starts to propagate, $\dot{M}_{\mathrm{tr}}$ would then have to be reduced by an extremely large factor; we made several numerical experiments with various prescriptions for a change in $\dot{M}_{\text {tr }}$ (amplitude and possible delay with respect to the formation of a cooling front), and we were unable to find a case in which the minimum disc radius was not obtained shortly after the end of a superoutburst.

\section{Possible solutions}

\subsection{ER UMa supercycles}

The standard TTI model cannot reproduce the very short supercycles observed in ultra-low mass-ratio systems. Osaki (1995a) reproduced RZ LMI's supercycle by ending prematurely the superoutburst assuming $r_{\text {crit0 }}=$ $0.42 a$ instead of the usual value of $0.35 a$ (note that in the TTI framework, one assumes $r_{\text {crit0 }}=0.35 a$ when modelling WZ Sge, even though its mass ratio is presumably smaller than in most SU UMa stars). In addition, the presence of superhumps after the end of the superoutburst implies that the eccentricity stops much later than the end of a superoutburst. The only ways for the disc to remain eccentric after the end of a superoutburst are then: (i) $t_{\mathrm{f}}$ is very long, or (ii) the accretion disc is always eccentric in ER UMa systems.

In the first case, the transition time $t_{\mathrm{f}}$ must be much larger than the one used here. However, as shown in Fig. 3, 
the interval between a superoutburst and the next normal outburst is far too long compared to observations. The second solution implies that the tidal torque is no longer responsible for superoutbursts, since $c(r)=c_{1}$ is a constant. However, the presence of large outbursts is still possible, as the (modified) DIM predicts the alternation of narrow and wide outbursts (see Paper I) for large enough mass transfer rates. If the mass transfer rate is constant, the light curve consists of one or two small outbursts surrounded by large ones, but small variations of the mass transfer rate can easily lead to ER UMa type light curves, provided that these variations show some regularity. Irradiation of the disc and of the secondary can also account for the presence of long and short outbursts; Hameury et al. (2000) have included these effects in the standard disc instability model and produced light curves typical of systems such as RZ LMi.

We therefore conclude that ER UMa stars should be dwarf novae with a permanently eccentric accretion disc, thereby accounting for superhumps, and where the illumination of the disc and the secondary star plays an important role. We thus predict that superhumps should exist at all phases of the supercycle of ER UMa stars; this apparently agrees with observations (Gao et al. 1999).

\subsection{Echo outbursts in WZ Sge stars}

WZ Sge stars have very long supercycles and superoutbursts. In addition, no normal outbursts between two consecutive superoutbursts are observed. Some WZ Sge stars also show echo outbursts at the end of the superoutburst: several small outbursts, spaced every tens of days, during which superhumps are still present.

Smak (1993) deduces the mass transfer rate of WZ Sge from the luminosity of the hot spot. It is observed to increase at least by a factor 10 during superoutburst, and it decreases afterwards, remaining larger than the quiescent value during several tens of days. Such mass transfer rate fluctuation could result from irradiation of the secondary star by the white dwarf. Hameury et al. (2000) have shown that irradiation (including disc irradiation) could indeed account for some peculiarities of WZ Sge stars: for example, they reproduced the echo outbursts phenomenon without including the tidal instability in the DIM; however, they did not reproduce a full cycle with long recurrence times, and the echo outbursts they obtained were slightly too dim.

The long recurrence times can be due to low alpha value (Smak 1993; Osaki 1995b), possibly due to a decay of the MHD turbulence that would lead to a timedependant $\alpha$-prescription (Osaki et al. 2001), thereby explaining the echo outbursts. A low viscosity could result from the secondary being a brown dwarf (Meyer \& Meyer-Hofmeister 1999), but one would have to explain why the viscosity is so much lower in these systems as compared to other SU UMa systems which have comparable or even shorter orbital periods. Another possibility
(Lasota et al. 1995, 1999; Hameury et al. 1997) is that WZ Sge stars are in a stable low state between superoutbursts, thus explaining the absence of normal outbursts. This requires a hole in the central regions of the disc, as a result of either a moderate magnetic field, or of evaporation. The superoutbursts would then be triggered by an externally imposed increase of the mass transfer rate, the long duration of the outburst and the large mass accreted onto the white dwarf being due to the irradiation of the secondary. They did not include the tidal instability and, as in the case of ER UMa stars, the presence of superhumps and late superhumps is explained if the accretion disc is always eccentric. If one combines these results with those of Hameury et al. (2000) on the echo outburst, one should be able to reproduce a WZ Sge star light curve with echo outbursts, which would however be slightly different than the observed ones.

We have used our TTI model including irradiation and the presence of an inner hole for the accretion disc as determined by Eq. (7) of Hameury et al. (2000). We use the parameters of EG Cnc. The mass transfer rate from the secondary is assumed to be affected by irradiation according to:

$\dot{M}_{\mathrm{tr}}=\max \left(\dot{M}_{2}, \gamma<\dot{M}_{\mathrm{acc}}>\right)$

where $\dot{M}_{2}$ is the mass transfer rate that would be obtained in the absence of illumination, $\gamma$ is a constant in the range [0-1], and $<\dot{M}_{\text {acc }}>$ is some average of the mass accretion onto the white dwarf, defined as:

$<\dot{M}_{\mathrm{acc}}>=\int_{-\infty}^{t_{0}} \dot{M}_{\mathrm{acc}} \mathrm{e}^{-\left(t_{0}-t\right) / \Delta t} \mathrm{~d} t$

$t_{0}$ being the actual time. This prescription differs slightly from that of Hameury et al. (1997), in that the average of the mass accretion rate is used instead of its value at time $t_{0}$; this accounts for the fact that a fraction of the accretion luminosity is released at later times when the white dwarf cools. In the following, we have taken $\Delta t=$ 14 days. We start with a stable system in the low state with a mass transfer rate of $1.5 \times 10^{15} \mathrm{~g} \mathrm{~s}^{-1}$, in agreement with Smak's (1993) estimate for WZ Sge $\left(2 \times 10^{15} \mathrm{~g} \mathrm{~s}^{-1}\right)$. At $t=10$ days, the mass transfer rate $\dot{M}_{2}$ is increased to $3 \times 10^{16} \mathrm{~g} \mathrm{~s}^{-1} ; 20$ days later, $\dot{M}_{2}$ is reduced to its initial value. Our results are insensitive on how $\dot{M}_{2}$ returns to its normal value, because illumination dominates in Eq. (4).

This model predicts that the luminosity should increase just before the triggering of a superoutburst. The increase need not be large - one simply requires the mass transfer rate to exceed the critical rate for stability; this therefore does not contradict the observation of Ishioka et al. (2002) that no strong orbital hump due to a high mass transfer rate was detected during the early phase of WZ Sge 2001 outburst.

We obtain a light curve with a very long superoutburst followed by 3 normal outbursts that last $\sim 3$ days and occur within two months of the main outburst (Fig. 5). The disc radius remains always larger than $0.35 a$, and is always 


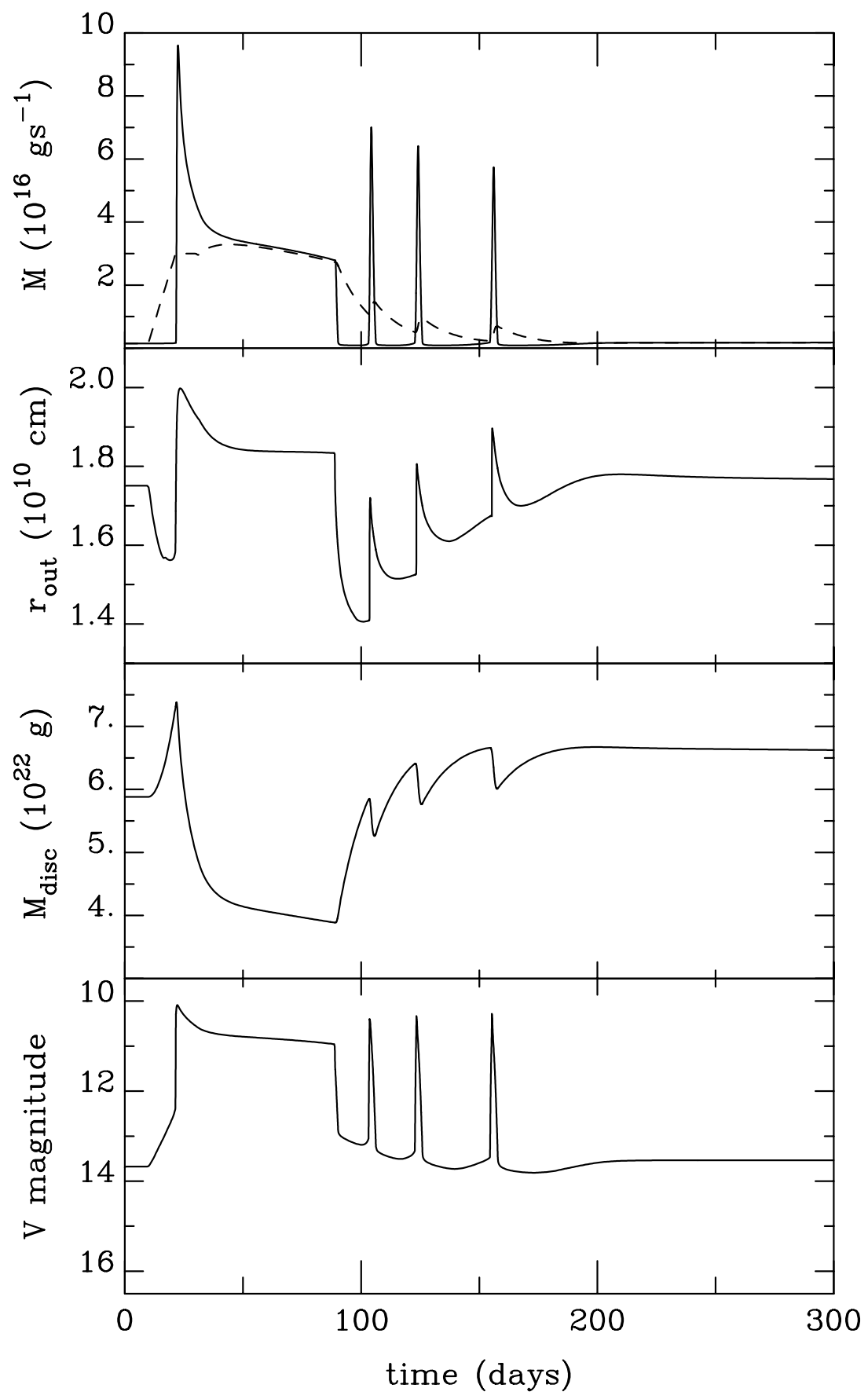

Fig. 5. Response of a steady accretion disc to a mass transfer rate variation by a factor 20 . We use the parameters of EG Cnc, include irradiation, the presence of a hole in the disc, and the existence of a tidal instability. Initially $\dot{M}_{2}=1.5 \times 10^{15} \mathrm{~g} \mathrm{~s}^{-1}$; at $t=10, \dot{M}_{2}$ increases up to $3.0 \times 10^{16} \mathrm{~g} \mathrm{~s}^{-1}$, and at $t=30$ returns to its quiescent value. The top panel shows the mass accretion rate onto the white dwarf (solid line) and the mass transfer rate from the secondary (dashed line); the other panels show the outer disc radius, its mass and visual magnitude.

in an eccentric state; permanent superhumps are thus expected. However, the interval between normal outbursts is twice longer than for EG Cnc echo outbursts, which are brighter than observed. We did not obtain a better fit with ER UMa light curves; in view of the very crude assumptions made to derive the mass transfer rate from the secondary under the influence of illumination, this is not really a surprise.

\section{Conclusion}

Hellier (2001) suggested that several limitations of the TTI model could be overcome if, in ultra-low mass ratio systems, the thermal instability ends while the disc is still eccentric. This would explain the late superhumps, and Hellier (2001) also suggested that this would account for the very short ER UMa supercycles and the WZ Sge stars' echo outbursts. 
We do find that the decoupling of the two instabilities can happen much more easily in ULMR systems than in other SU UMa stars. However, the tidal instability stops almost immediately after the end of the thermal-viscous instability (delay less than a day), because the disc shrinks very rapidly after the departure of a cooling wave. We estimate that it takes only a few days for the disc to return to a circular shape after the cessation of the tidal instability. This is still insufficient to explain late superhumps that are found tens of days after the superoutburst in some systems.

We propose instead that the discs in ER UMa systems are permanently eccentric, and that they should therefore have permanent superhumps; there are observational indications of this (Gao et al. 1999). The short supercycle is then due to either an alternation of narrow and wide outbursts as in SS Cyg (see Buat-Ménard et al. 2001), or to the effects of irradiation (see Hameury et al. 2000). Further observations of late superhumps should confirm their permanence in ER UMa stars.

As in Hameury et al. (1997), we propose that WZ Sge stars are in a stable cold state during quiescence. The superoutburst is then triggered by an enhancement of mass transfer rate, and echo outbursts are triggered while the mass transfer rate is still high after the superoutburst. However, simulated light curves are still somewhat different from observations; this might be due to our crude treatment of the secondary illumination.

Acknowledgements. This work was supported in part by a grant from Programme National de Physique Stellaire of the CNRS. We thank J. P. Lasota for a very careful reading of the manuscript and for helpful comments. We are grateful to the referee, Prof. Y. Osaki, for pointing out an error in one of the sets of parameters used.

\section{References}

Buat-Ménard, V., Hameury, J.-M., \& Lasota, J.-P. 2001, A\&A, 366,612
Gao, W., Li, Z., Wu, X., Zhang, Z., \& Li, Y. 1999, ApJ, 527, L55

Hameury, J.-M., Lasota, J.-P., \& Huré, J.-M. 1997, MNRAS, 287, 937

Hameury, J.-M., Menou, K., Dubus, G., Lasota, J.-P., \& Huré, J.-M. 1998, MNRAS, 298, 1048

Hameury, J.-M., Lasota, J.-P., \& Warner, B. 2000, A\&A, 353, 244

Hellier, C. 2001, PASP, 113, 469

Ichikawa, S., Hirose, M., \& Osaki, Y. 1993, PASJ, 45, 243

Ishioka, R., Uemura, M., Matsumoto, K., et al. 2002, A\&A, 381, L41

Kornet, K., \& Różyczka, M. 2000, Acta Astron., 50, 163

Kuulkers, E. 1999, in Disk Instabilities in Close Binaries 25 years of the Disk Instability Model, ed. S. Mineshige, \& J. C. Wheeler (Universal Academy Press, Tokyo), 169

Lasota, J.-P. 2001, New AR, 45, 449

Lasota, J.-P., Hameury, J.-M., \& Huré, J.-M. 1995, A\&A, 302, L29

Lasota, J.-P., Kuulkers, E., \& Charles, P. 1999, MNRAS, 305, 473

Meyer, F., \& Meyer-Hofmeister, E. 1999, A\&A, 341, L23

Murray, J. R. 1996, MNRAS, 279, 402

Osaki, Y. 1989, PASJ, 41, 1005

Osaki, Y. 1995, PASJ, 47, L25

Osaki, Y. 1995, PASJ, 47, 47

Osaki, Y., \& Meyer, F. 2002, A\&A, 383, 574

Osaki, Y., Meyer, F., \& Meyer-Hofmeister, E. 2001, A\&A, 370, 488

Papaloizou, J., \& Pringle, J. E. 1977, MNRAS, 181, 441

Patterson, J., Kemp, J., Jensel, L., et al. 2000, PASP, 112, 1567

Semeniuk, I. 1980, A\&AS, 39, 29

Shakura, N. I., \& Sunyaev, R. A. 1973, A\&A, 24, 337

Smak, J. 1984, Acta Astr., 34, 161

Smak, J. 1993, Acta Astr., 43, 101

Stehle, R. 1999, MNRAS, 304, 687

van Paradijs, J. 1983, A\&A, 125, L16

Warner, B. 1995, Cataclysmic variable stars (Cambridge University Press, Cambridge)

Whitehurst, R. 1988, MNRAS, 232, 35 